Classification

Physics Abstracts

$78.90 \mathrm{t}-81.70 \mathrm{Dw}-87.45 \mathrm{k}$

\title{
Scanning probe microscopy: applications in biology and physics
}

\author{
Othmar Marti, Jaime Colchero, Hartmut Bielefeldt, Michael Hipp and Achim Linder
}

Fakultät für Physik, Universität Konstanz, D-78434 Konstanz, Germany

(Received August 31 1993; accepted September 29, 1993)

\begin{abstract}
Scanning probe microscopes can probe a variety of quantities characterizing surfaces. This overview paper describes techniques applicable in an ambient environment and having the power to distinguish different materials: the scanning force and friction microscope and the scanning nearfield optical microscope combined with a spectrometer. The basic operating principles of these two microscopes are described. Selected experiments point to possible future applications: we discuss scanning force and friction microscopy of $\mathrm{ZnSe}$ on $\mathrm{GaAs}$ and of $\mathrm{Na}, \mathrm{K}$-ATPase and near-field optical microscopy of a grating and of micropores.
\end{abstract}

\section{Introduction.}

Scanning probe microscopy techniques [1] are powerful tools to investigate atomic and molecular scale objects in biology and physics. Various kinds of interactions are employed, such as electron tunneling [2], attractive [3], repulsive [4] and frictional forces [5], and optical effects [6]. Due to the inherent nonlinear interaction characteristics scanning probe microscopes can be used to trigger events or modify surfaces or objects [7]. Unique to the scanning probe microscopy techniques is the wide range of operating environments [1], comparable only to classical optical microscopy. Experiments are now routinely performed in air, liquids and in ultra high vacuum.

Many samples should be investigated under ambient conditions, especially in biology or in application relevant fields. It is desirable that a microscope measures not only the topography of a sample, but also additional quantities giving a detailed view of the composition of the sample. At length scales larger than a micrometer, optical spectroscopy is well suited for this task [8]. For operation in vacuum conditions many techniques with this capability exist, such as Auger electron spectroscopy, photo electron spectroscopy and others [9].

Conductive samples can be investigated down to the atomic scale at ambient conditions or in electrochemical environments by scanning tunneling microscopy and spectroscopy [10]. However, the vast majority of surfaces is isolating: therefore scanning tunneling microscopy is of only limited use for real sample surfaces, such as biological samples or samples of technological relevance.

Scanning force microscopy is a well proven technique which allows the measurement of the topography of isolating and conductive samples down to the atomic scale [1]. By measuring not 
only the normal force but also lateral forces one is able to calculate friction coefficients [11]. Using this information materials with different friction coefficients [12] can be distinguished. However the friction coefficient is not a quantity characterizing a material uniquely. Therefore it is not possible to label an unknown material using only the friction information.

Optical spectroscopy [8] has the capability to positively identify bonds and bond configurations in organic and polymer materials. By combining optical spectroscopy with a sub-wavelength sized light emitter it is possible to image samples with a $50 \mathrm{~nm}$ lateral resolution [6]. Additionally optical detection is inherently much faster than force microscopy. The width of the optical spectrum allows a much broader information to be gained from a sample than would be possible by scanning force and friction microscopy. Techniques such as fluorescence or photoluminescence microscopy guarantee that the information is virtually free from interference from other processes [8].

\section{Common Principles of Scanning Probe Microscopes.}

In classical physical experiments one tries to decouple the probe from the sample and the processes occurring in the sample. The interaction of the probe should have only vanishing influence on the sample. A practical limit of the lateral resolution exists for information transported by waves, such as light or in a quantum mechanical sense, electrons. Two objects arranged perpendicularly to the direction of propagation of the waves can only be distinguished in the far field if they are separated by more than $\lambda / 2$, where $\lambda$ is the wavelength of the interaction (Fig. 1). This law due to Abbe is a practical statement: Two objects with a separation much smaller than $\lambda / 2$ do create wave components which carry the information about their separation. However the amplitude of these wave components vanishes much faster with distance than the propagating modes. A detector very close to the two objects senses these near-field components and can distinguish objects that are closely spaced. The size of the detector must be smaller than the desired resolution and, most importantly, the distance between the detector and the objects must be very much smaller than the size of the detector.

Far-field methods mostly acquire an entire image at one time, allowing very short acquisition times. Near-field or proximity methods on the other hand, do only measure quantities from a very small spot. Therefore a surface must be scanned to get an image of the surface. Consequently proximity methods are slow. In addition due to the small spacing between the detector and the sample, the coupled system detector-sample must be taken into account for calculations of the interaction. Since the coupling between the probe tip and the sample is nonlinear, the same probe and interaction can be used to trigger events or processes and to monitor them.

The resolution of a scanning probe microscope is determined by the size of the probe, the distance between the probe and the sample, and by the decay characteristics of the interaction [1]. Figure 2a, shows the effect on the resolution by assuming different power laws of the interaction. For this calculation we assume two point like sources of an interaction located at -10 and at +10 . A square detector with a size of 2 by 2 is scanned across the sample surface at a distance of 20 . The different lines show the shape of the measured signal as a function of the position of the detector for interactions having a power law with powers ranging from -1 to -8 . All curves are scaled to the maximum intensity on the detector due to one source. It is obvious from this figure that steeper decays produce a better resolution. This is one of the reasons, why non-contact force microscopy tends to have a worse resolution than contact force microscopy.

Figure $2 b$, shows the effect of changing the distance between the probe tip and the sample. The same setup of two point like sources at -10 and +10 is used. The power law of the test interaction is set to a fixed exponent of -3 , the size of the detector is the same as in figure 2a. All curves are scaled to the maximum intensity on the detector due to one source. The height varies from 1 to 
Far Field Methods

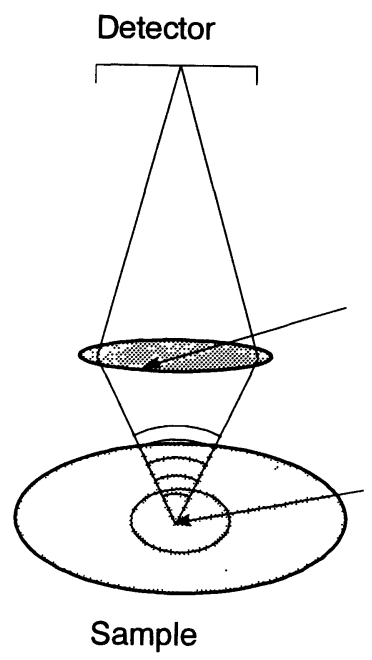

Scanned Probe Methods

Transmission

(Imaging)

System

Interaction

(Characteristic Length $\lambda)$

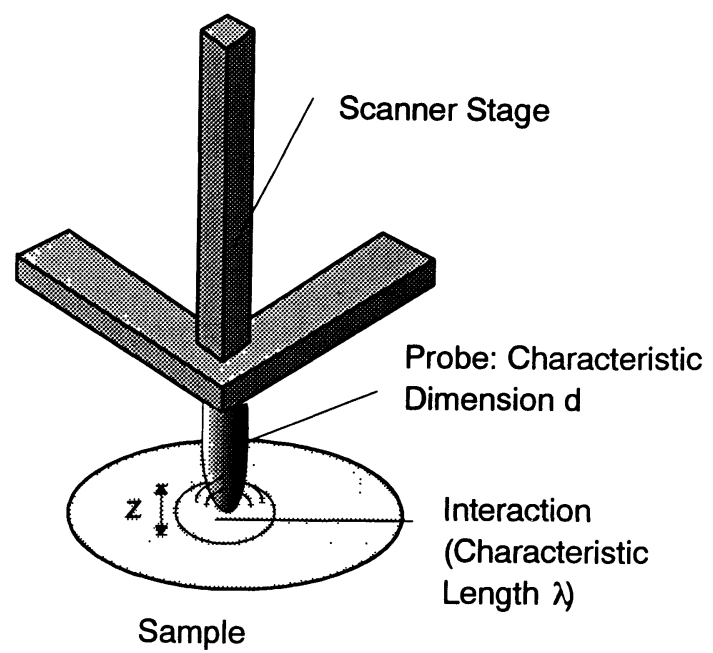

$\mathrm{Z}, \mathrm{d}<\lambda$

Fig. 1. - The left side shows a schematic view of a far field experiment, consisting of an interaction region, a transmission path and a detector. The right side shows a proximity detection setup. The probe and sample are closely spaced and their states are coupled by the interaction. The probe is scanned by the scanner stage.

64. This figure shows, that the resolution is a function of the separation between the detector and the sample.

Hence the conlusion can be drawn, that the distance between the probe and the sample should be as small as possible, independent of the kind of probe or interaction used.

\section{Friction measurements.}

Friction force microscopy allows to classify materials by their friction coefficient [12]. In this chapter we briefly discuss our setup, introduce the two-dimensional histogram technique and show some results on $\mathrm{Na}$, K-ATPase $[13,14]$ and $\mathrm{ZnSe}$.

3.1 THE FRICTION MICROSCOPE. - Figure 3 shows a sketch of our scanning force and friction microscope based on the optical lever principle[15]. Light from a laser diode is reflected off the surface of a cantilever. The orientation of the reflected light is determined by a four-segment photo diode. A change of the direction of the reflected light corresponds to a change of the surface normal of the cantilever beam. This change is due to several quantities: first, forces normal to the sample surface will bend the cantilever upwards or downwards, depending on the sign of the force. Second, lateral forces along the cantilever axis will induce a similar bending of the cantilever. However, for most setups this force can be neglected. Third, lateral forces perpendicular to the cantilever axis will twist the cantilever, because of the torque applied.

A detailed description of the functioning of the scanning force and friction microscopes can be found in the literature [1]. 

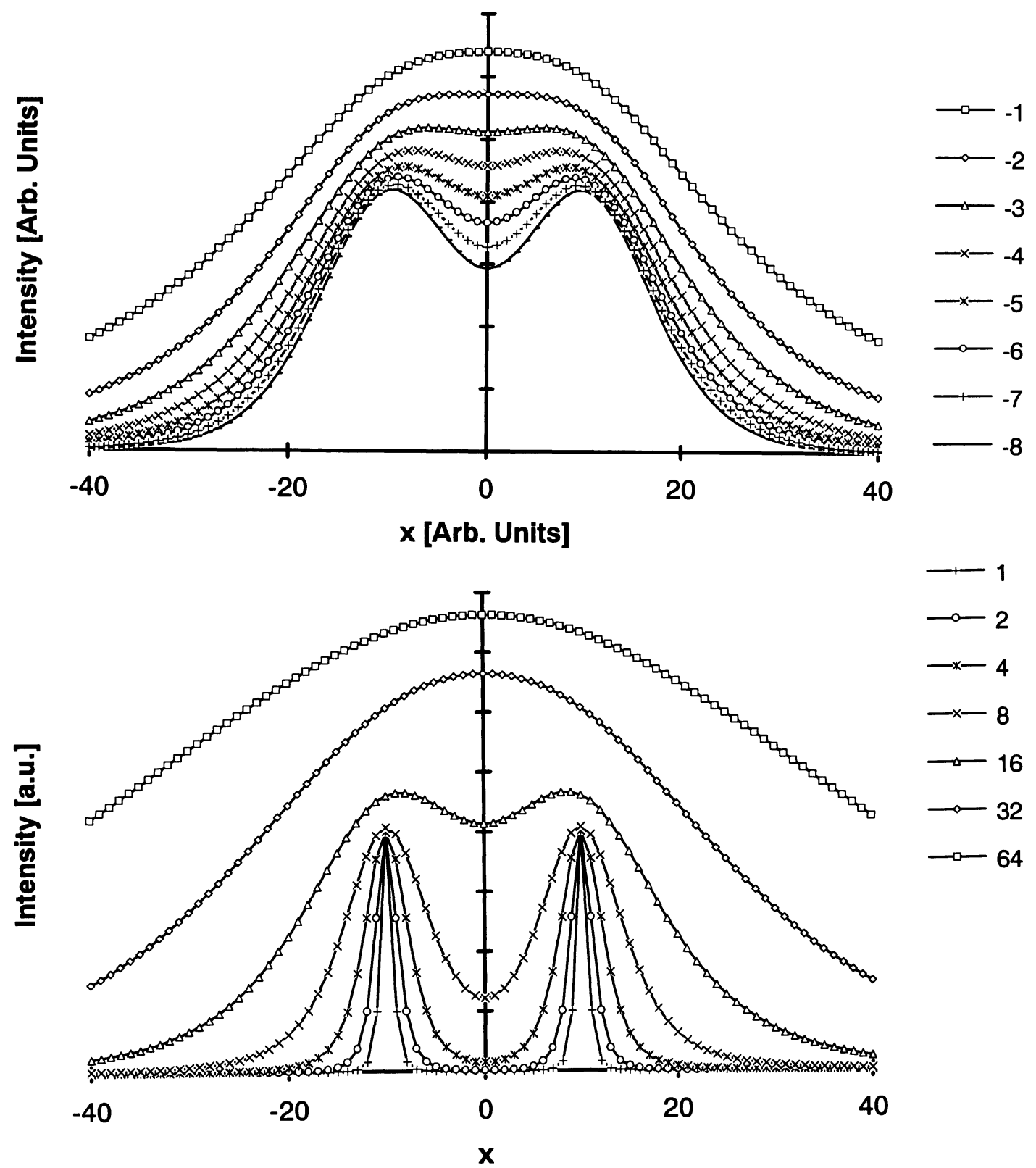

Fig. 2. - Resolution in the proximity mode. a) shows the expected signal when the power law of the interaction is changed. The numbers labelling the curves are the exponents in the power law. b) shows the effect of changing the separation between the probe and the sample. The separation in arbitrary units is used as a label for the curves.

3.2 HistogRAMS. - The friction coefficient of a pair of materials may be seen as a correlation coefficient between two forces. A scanning force and friction microscope imaging simultaneously the normal and lateral force components determines a measure of the correlation between the forces. To visualize this correlation two dimensional histograms are best suited [12]. As an ex- 


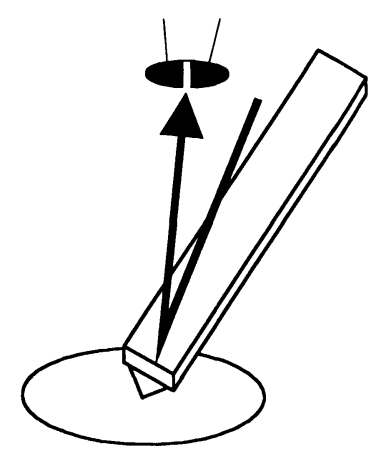

a)

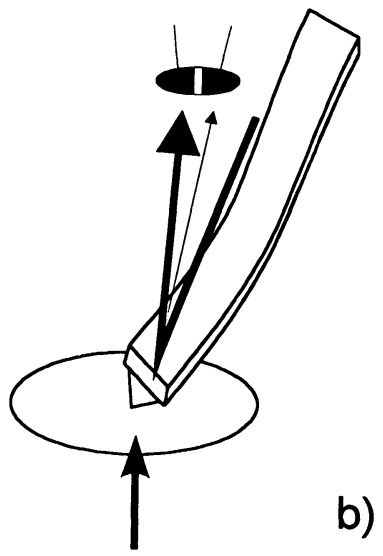

b)

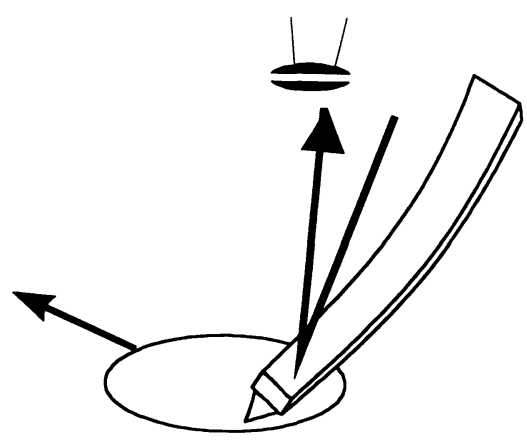

c)

Fig. 3. - Light is reflected from a cantilever and the position on the detector is measured a). A force normal to the sample surface deflects the cantilever upwards and the light beam to one side b). Moving the sample induces a torsion in the cantilever. The light beam is deflected perpendicular to $b$ ).

ample figure 4a shows two signals mimicking two different physical quantities which are perfectly correlated. The curve with squares is the sine function; the curve with the diamond symbol is the cosine function. Every corresponding pair of data points one defines one point in a plane, for instance with the amplitude of the sine defining the horizontal axis. This plane is now divided into rectangular bins. For each bin we count the number of data points falling into it. This number is then interpreted as the height of a surface over the parameter plane. Similar to the rendering of scanning probe microscope data the number of points falling into the spatially distributed bins is plotted as a function of the position of the bin. In our example the correlation of sine and cosine is a circle, as expected (Fig. 4b).

This two-dimensional histogram procedure can now be applied to the normal and lateral force in a force microscope image of $\mathrm{ZnSe}$. Figure 5 shows the measured data together with the 2dimensional histogram. The slope of the data points cloud is the friction coefficient. On inhomogeneous samples this type of analysis can yield the different friction coefficients when different materials are present. 


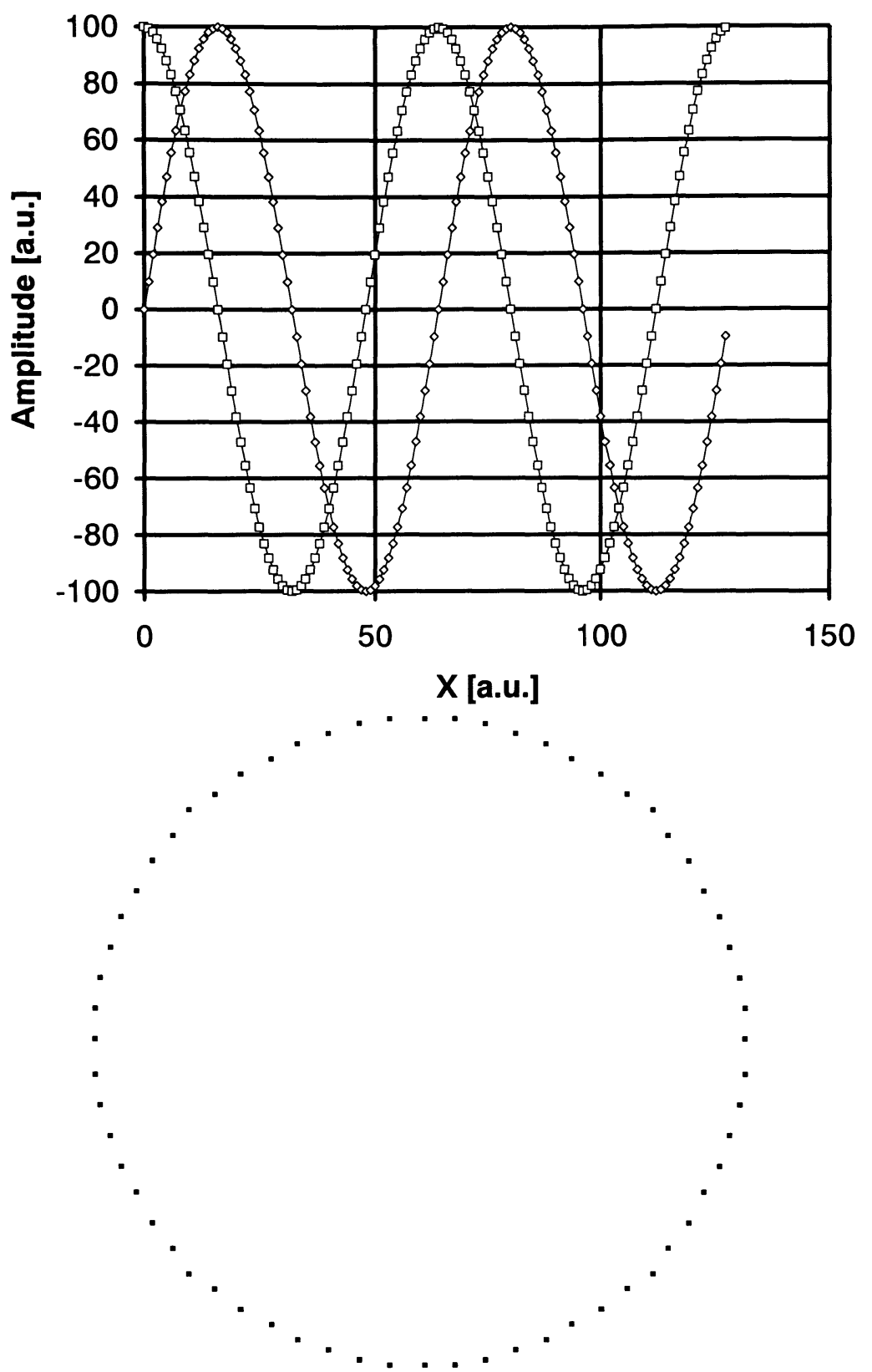

Fig. 4. - a) shows two correlated signals, a sine and a cosine. The two-dimensional histogram of these two correlated signals is shown in part $b$ ).

3.3 $\mathrm{Na}, \mathrm{K}$-TPASE. - The scanning friction microscopy of $\mathrm{Na}$, K-ATPase yields interesting results on the interaction of the tip with lipid bilayers which do and do not contain proteins [13, 14]. The preparation of the cell membrane fragments is standard and described elsewhere [16]. The fragments are typically imaged in water with an imaging force of about $1 \mathrm{nN}$ (Fig. 6a and b). In this case individual Na, K-ATPase-dimers with a size of $10 \times 12 \mathrm{~nm}$ can usually be observed. Fig- 

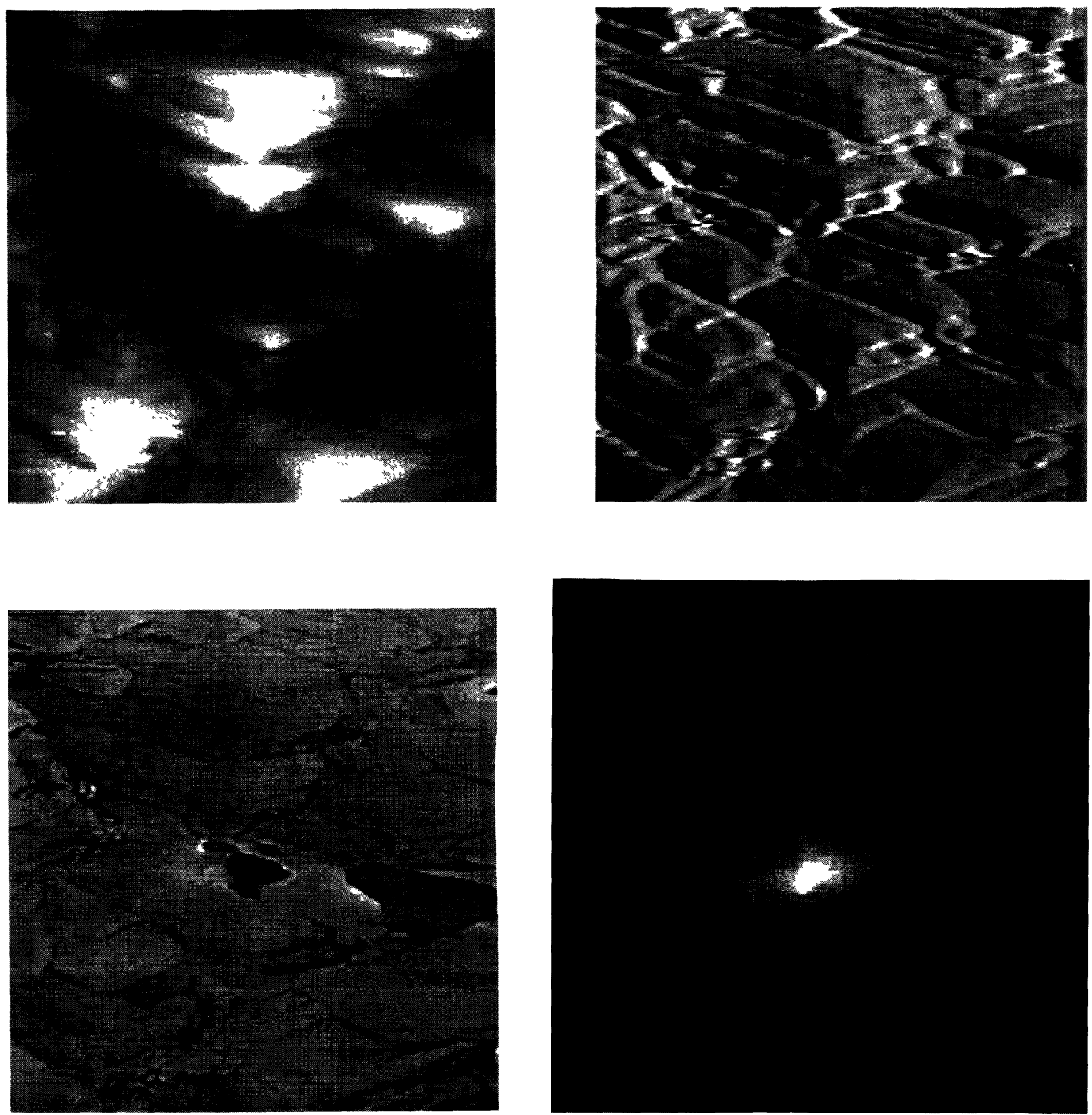

Fig. 5. - a) shows the topography of a $\mathrm{ZnSe}$-surface. The size of the image is $3 \mu \mathrm{m}$ by $3 \mu \mathrm{m}$. The height ranges from 0 (black) to $110 \mathrm{~nm}$ (white). b) shows the normal force, c) the friction force and d) the friction coefficient, as determined by a two-dimensional histogram.

ure 6 shows a combined topographic and lateral force image of a membrane fragment at medium resolution. The topographic image shows two distinct domains on the fragment, a lower one (height: $4 \mathrm{~nm}$, left part of the fragment) with corresponds to the lipid domain and a higher one corresponding to the protein domain (height: $12 \mathrm{~nm}$, individual proteins are not resolved at this resolution). The most evident feature in the lateral force images is the inhomogeneity of the substrate. The lateral force is low on the fragment as well as on the debris which partly covers the substrate. We find lateral forces ranging from $40-25 \mathrm{nN}$ on the dark regions of the substrate and up to about $3 \mathrm{nN}$ on the protein phase of the membrane fragments. Within our detection limit (1 $\mathrm{nN}$ for lateral forces) we could not detect any lateral forces on the lipid phase of the membrane 
fragment. Vanishing lateral forces have been observed also on some areas of the protein phase. This is probably due to soft adsorbed material. Some of the bright structures on the substrate also appeared in the topographic images with a corrugation between 2 and $4 \mathrm{~nm}$; therefore we think that these structures were composed of lipid debris from preparation. Lateral forces were surprinsingly high compared to the low imaging force $(<2 \mathrm{nN})$. Under standard conditions force normal to the surface did not exceed about $5 \mathrm{nN}$, even if the additional adhesion force is taken into considerations. The lateral forces on the substrate are higher at least by a factor of four. Since SFM and SFFM techniques are a very new field, no additional data of SFFM in electrolytes are available for comparison at the moment.
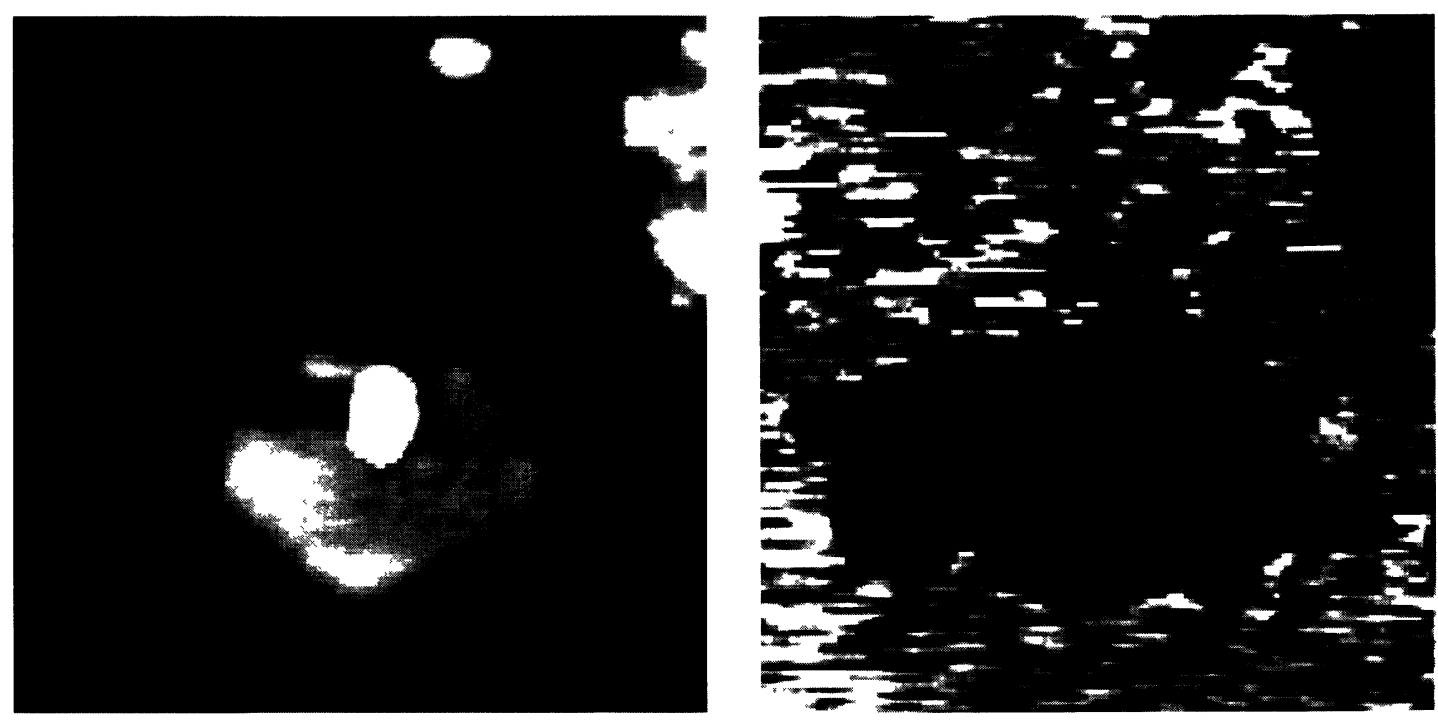

Fig. 6. - Topographic- (left) and lateral force (right) image of a membrane fragment containing Na, KATPase. The image size is $1.5 \times 1.5 \mu \mathrm{m}^{2}$. The gray-scale corresponds to a corrugation of about $20 \mathrm{~nm}$ (left) and a lateral force of about $40 \mathrm{nN}$ (right). The imaging force was $2 \mathrm{nN}$. In the topographic image two different domains are clearly resolved: a lower, flat one of about $4 \mathrm{~nm}$ height and a higher, structured domain of about $12 \mathrm{~nm}$ height. The first domain is composed only of lipids, whereas the second domain contains the proteins.

\section{Near field optical microscopy and spectroscopy.}

The scanning force and friction microscope does not have the capability of uniquely determining an unknown substance on the sample surface. This is because friction coefficients are not unique. Optical spectroscopic methods on the other hand allow a detailed investigation of absorption and fluorescence which can lead to a positive identification of an unknown substance.

4.1 EXPERIMENTAL SETUP. - The main idea behind any near field optical microscope is to have a source or collector of light with a size smaller than the sought resolution [17]. We have chosen sharpened fibers as a detector, mainly because they are easily handled and because fiber optics 
can be used to interface to other components. The schematic setup of our spectrometer is shown in figure 7 [18]. The light collected by the fiber is sent through a fiber coupler and then analyzed in a spectrometer. The output of this spectrometer is summed up over a small, selectable frequency band. At the input of the spectrometer a small fraction of the total intensity is deflected to a second detector measuring the integral intensity. Both signals are recorded by a computer based data acquisition system. The distance between the tip and the sample is controlled by a shear force measurement [19]. The sample can be illuminated either through the fiber, in an evanescent geometry or by a global illumination of the sample. Illumination through the fiber is achieved by coupling light into an input arm of the fiber coupler. Evanescent illumination is set up by letting light reflect totally at a glass air interface. This setup allows for virtually all types of measurements which can be done by conventional optical microscopy.

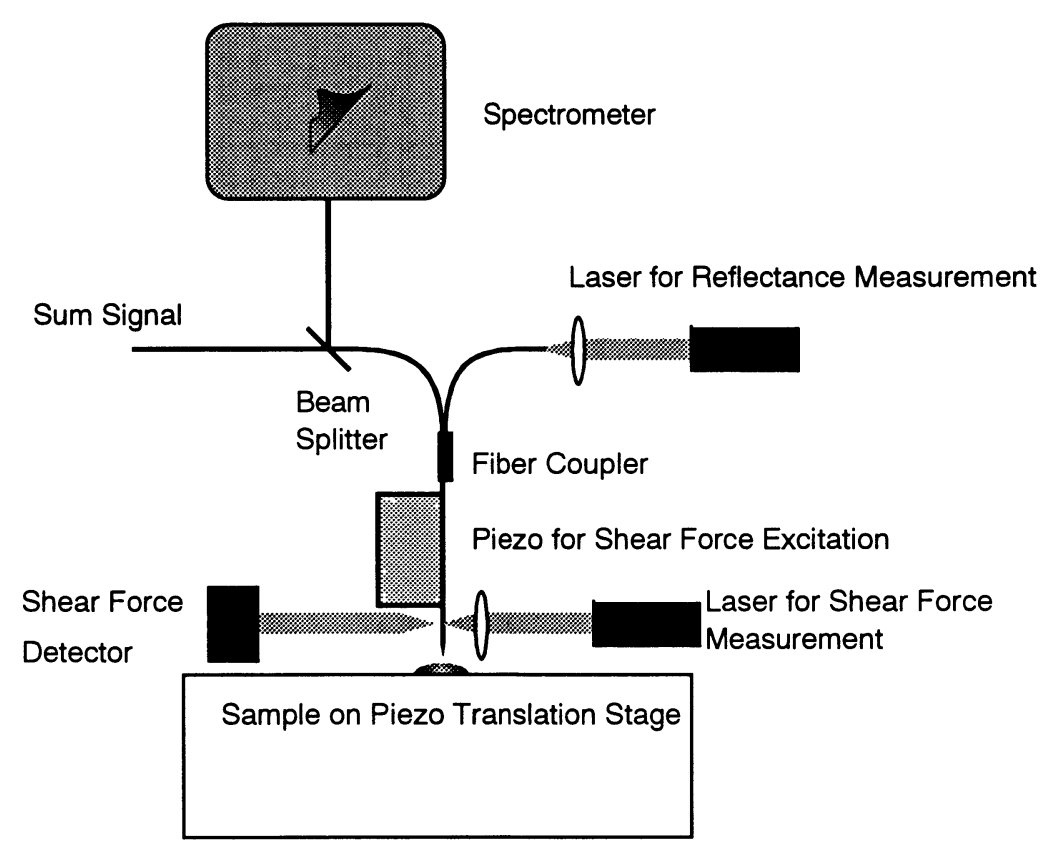

Fig. 7. - The basic setup of a scanning near-field optical microscope with shear force detection.

4.2 RESULTS. - A first example of near-field microscopy is the imaging of an optical grating. Figure $8 \mathrm{a}$ shows the topography, as measured by shear force, and in figure $8 \mathrm{~b}$ the reflected intensity at $670 \mathrm{~nm}$. The grating has a period of $415 \mathrm{~nm}$, which is faithfully recovered. On most of the data set the reflectance signal varies proportional to the surface topography. However, on the upper left side there is a feature in the topography which does not appear in the reflectance signal.

In general the reflectance signal is, besides the reflectance, dependent on the orientation of the sample. There are, however, examples where this is not the case. Such an example is shown in figure 9a. Figure 9a shows the topography and figure $9 \mathrm{~b}$ the reflection coefficient of a filter with nanometric pores [20]. The filter has a distribution of holes all with a nominal thickness of $1 \mu \mathrm{m}$. The reflection signal shows a reproducible granularity of about $1 \mu \mathrm{m}$. This is consistent with a tip radius of $1 \mu \mathrm{m}$, as determined from the data and also from SEM images made of the tip after use. 

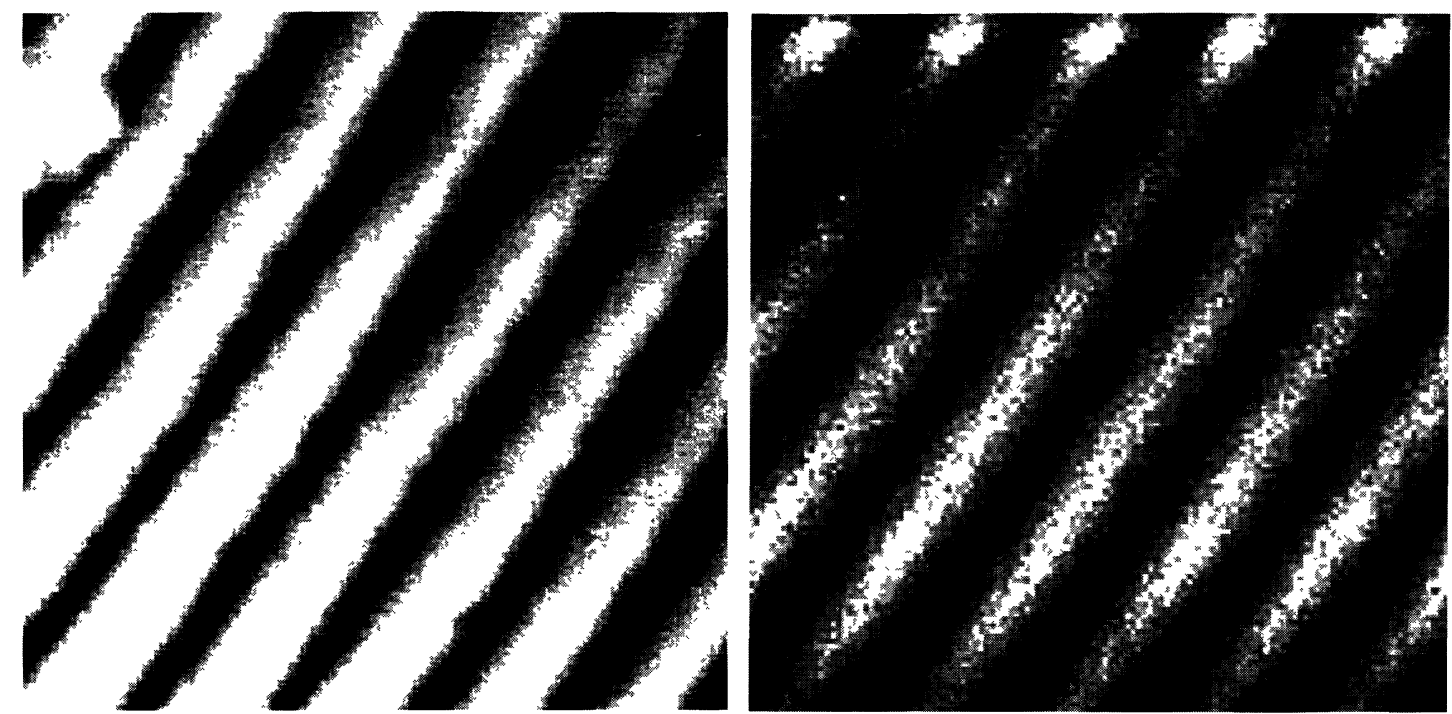

Fig. 8. - a) shows the topography of an optical grating as measured by the scanning near-field optical microscope. b) is the reflected light at $670 \mathrm{~nm}$. The image size of the image is $2.4 \mu \mathrm{m}$ by $2.4 \mu \mathrm{m}$. The height varies from 0 to $5 \mathrm{~nm}$.
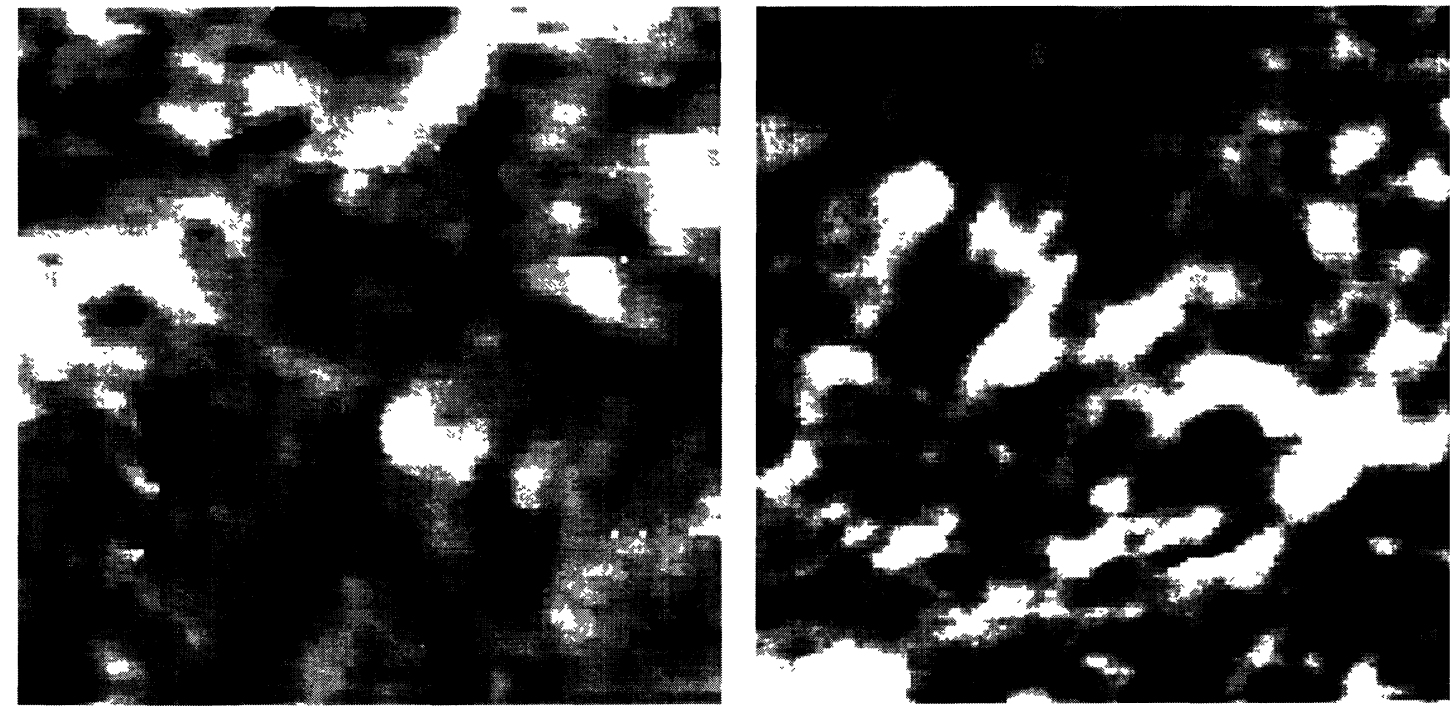

Fig. 9. - a) shows the topography of a filter membrane with $\mu \mathrm{m}$-sized pores. b) is the reflected light at $670 \mathrm{~nm}$. The image size is $11 \mu \mathrm{m}$ by $11 \mu \mathrm{m}$. The height varies by $10 \mathrm{~nm}$. 


\section{Conclusions.}

Scanning force and friction microscopy as well as scanning near-field optical microscopy are tools which allow to distinguish different materials at ambient conditions. Unlike conventional high resolution microscopes with a material contrast these two techniques can be operated in air or in liquids. Scanning force and friction microscopy is the tool of choice when highest resolution is demanded. Some prior knowledge about the sample is required, though.

Near field optical microscopy on the other hand does not have such a high spatial resolution. Its strength are the well known optical methods which can be applied at very small areas. There is, however, a tradeoff between highest resolution and highest signal to noise ration. The smaller the fiber tip becomes, or the smaller the relevant aperture is, the fewer photons are transmitted to the detector. Therefore one must balance the demands for high resolution and high signal to noise ratio.

Near field optical microscopy also has the potential to achieve very high time resolution on systems which can be periodically excited through the use of pump-probe techniques.

Scanning force and friction microscopy and scanning near-field optical microscopy will be applied to real world problems in manufacturing environments.

\section{Acknowledgements.}

The authors would like to thank J. Mlynek for his continuing support of their work. We thank H.-J. Apell, K. Dransfeld, R. Möller, E. Weilandt, J. Mertz and I. Hörsch for many useful discussions. We thank P. Pliska for the grating and D. Courjon and E. Ferain for the cyclopore filter. The funding of this research by the Deutsche Forschungsgemeinschaft through their various grants and by the Land Baden-Württemberg (Landesschwerpunkt) is gratefully acknowledged.

\section{References}

[1] MARTI O., "Scanning Probe Microscopy: an Introduction”, in O. Marti and M. Amrein, Eds. Academic Press San Diego, 1-126 (1993), and references therein.

[2] Binnig G., Rohrer H., Gerber Ch. and Weibel E., Phys. Rev. Lett. 49 (1982) 57.

[3] HORIE C. and MiYAZAKI H., Phys. Rev. B42 (1990) 11757.

[4] Binnig G., QuATE C.F. and Gerber Ch. Phys. Rev. Lett. 56 (1986) 930.

[5] Mate C.M., MCClelland G.M., Erlandsson R. and Chiang S., Phys. Rev. Lett. 59 (1987) 1942.

[6] DÜrig U., POHL D.W. and Rohner F., J. Appl. Phys. 59 (1986) 3318-3327.

[7] SChNeIR J., SonNenfeld R., MARTI O., HANSMa P.K., Demuth J.E. and Hamers R.J., J. Appl. Phys. 63 (1988) 717-721.

[8] DEMTRÖDER W., Laser Spectroscopy, (Springer Berlin, 1988).

[9] ZANGWILl A., Physics at Surfaces, (Cambridge University Press, 1988).

[10] SIEGENTHALER H. and CHRISTOPH R., Nato ASI (Kluwer) Ser. E 184 (1990) 242-267.

[11] MARTI O., COLCHERO J. and MLYNEK J., Nanotechnology 1 (1990) 141.

[12] MARTI O., Nanotribology: Friction on a Nanometer Scale, Physica Scripta, in press.

[13] APELl H.-J., COlChERO J., LINDER A., MARTI O. and MLYNEK J., Ultramicroscopy $42-44$ (1992) 11331140.

[14] Linder A., APELl H.-J., ColChero J. and MARTi O., "Na, K-ATPase: Preparation and Scanning Force Microscopy", in O. Marti and M. Amrein, Eds., "STM and SFM in Biology", Academic Press San Diego (1993) 275-308.

[15] HiPP M., BIELEFELDT H., COLCHERo J., MARTI O. and MYLNEK J. Ultramicroscopy 42-44 (1992) 14981503. 
[16] JøRGENSEN P.L., ANDERSEN J.P., J. Membr. Biol. 103 (1988) 95.

[17] POHL D.W., Adv. Opt. Electr. Micr. 12 (1991) 243-312;

E. Betzig, J.K. Trautman, Science 257 (1992) 189-195.

[18] BIELEFELDT H., HECHT B., LUX-STEINER M. and MARTI O., to be published.

[19] BetZig E., FinN P.L., WeINER J.S., Appl. Phys. Lett 60 (1992) 2484-2486.

[20] Filter with $1 \mu \mathrm{m}$ holes, $4 \times 10^{7}$ holes per $\mathrm{cm}^{-2}$, made by Cyclopore S.A., Louvain la Neuve, Belgium. 